\title{
Editorial
}

\section{Sensors for Robotics 2015}

\author{
Aiguo Song, ${ }^{1}$ Guangming Song, ${ }^{1}$ Daniela Constantinescu, ${ }^{2}$ Lei Wang, ${ }^{3}$ and Quanjun Song ${ }^{4}$ \\ ${ }^{1}$ Robot Sensor and Control Lab, School of Instrument Science and Engineering, Southeast University, Nanjing 210096, China \\ ${ }^{2}$ Department of Mechanical Engineering, University of Victoria, P.O. Box 3055, Victoria, BC, Canada STN CSC \\ ${ }^{3}$ School of Computer Science and Software Engineering, University of Wollongong, Wollongong, NSW 2522, Australia \\ ${ }^{4}$ Institute of Intelligent Machines, Chinese Academy of Sciences, Hefei 230031, China
}

Correspondence should be addressed to Aiguo Song; a.g.song@seu.edu.cn

Received 1 July 2015; Accepted 6 July 2015

Copyright (C) 2015 Aiguo Song et al. This is an open access article distributed under the Creative Commons Attribution License, which permits unrestricted use, distribution, and reproduction in any medium, provided the original work is properly cited.

Robot is currently one of the exciting and fast developing technologies changing the life of human being. It has been widely applied in lots of areas such as industry, agriculture, medicine, transportation, social service, military, space exploration, and undersea exploiting. Increasing attention by robot researchers has been paid to the robot sensor, as a key component of the robot. During the last decade, much effort has been done to develop robot sensors for robot perception, robot control, autonomous robot, human-robot interaction, and so forth. In spite of the large and increasing interest and promising applications, robot sensor design is a significant challenging, which is involved in not only sensor materials, structure design, manufacturing process, and calibration technique, but also signal processing, data fusion, and pattern recognition. For instance, remarkable examples of tactile sensors and systems have been proposed; however, their ability to address specific applications and their extension to other fields such as medical instrumentation, prosthetic devices, and biomechanics test is questionable.

This special issue aims at exhibiting the latest research achievements, ideas, and advances in robot sensors. The special issue summarizes the most recent developments in the field of sensors for robotics. The theme of 2015 special issue focuses on the robot force and tactile sensor, robot sensor fusion, and robot sensor applications.

Force and tactile sensors are absolutely necessary elements for robot when interacting with environment. The paper by A. Almassri et al. surveys the state-of-the-art in variety force sensors for designing and application of robotic hand. This paper introduces the different techniques for measuring force or interface pressures. These techniques include load cells, pressure indicating film, and tactile pressure system. Similarly, a review on industry pressure sensing that involves the pick and place applications and algorithm control is also highlighted. The paper also discusses the MEMs sensor technology and different types of sensors. At last, it discusses the piezoresistive flexiforce sensor. Flexiforce sensor has a good substrate material, which is a polymer that enhances the force sensing and improves the performance of force, linearity, hysteresis, drift, and temperature sensitivity compared to any other thin film. Furthermore, it is flexible and ultrathin enough so that it can be widely used as robot hand force sensor and tactile sensor. The paper by $\mathrm{C}$. Wu et al. introduces the application of tactile sensor in prosthetic hand. This paper proposes an EMG prosthetic hand control strategy using force sensor and tactile sensor to improve the control effectiveness and make the prosthetic hand not only controllable but also perceivable. The control strategy consists of EMG self-learning motion recognition, back stepping controller, and force tactile representation. The force and tactile information are not only used for hand grasp control but also for haptic stimulating on user, which helps the user perceive the states of the prosthetic hand.

Robots rely on multiple sensors to provide them with information about their surroundings. Thus, sensor fusion based robot sensing is always a key issue for object tracking, robot path plan and navigation, environment understanding, and autonomous behaviors. The paper by D. Tuvshinjargal et al. proposes a sensor fusion based reactive motion planning method for an autonomous vehicle in dynamic environments. The dynamic motion planning method combines the reactive motion planning technique with a sensor fusion 
based obstacle detection approach, which results in improving robustness and autonomy of vehicle navigation within unpredictable dynamic environments. The key feature of the motion planning method is based on a local observer in the virtual plane which allows the effective transformation of complex dynamic planning problems into simple stationary in the virtual plane, and a sensor fusion based obstacle detection algorithm provides the pose estimation of moving obstacles by using a Kinect sensor and a sonar sensor, which helps to improve the accuracy and robustness of the reactive motion planning approach in uncertain dynamic environments.

In the past decades, person tracking system using a robot has achieved a lot of improvements. However, the problems of distinguishing person and reliable following still exist. The paper by S. Jia et al. proposes a person detection and tracking method by representing a person with multicues based on patches and designing a fuzzy based intelligent gear control strategy (FZ-IGS). The person detection algorithm includes a detector and a tracker. The detector divides a person into many patches and represents a patch by the use of multicues including depth, color, and texture. As track evolves, the detector adjusts the person's size according to depth information. By analyzing the depth histograms and patches' similarity with the given person, the detector can easily recognize the occlusion and then make a decision to update the person's appearance model and change the tracking strategy. The tractor based on an extended Kalman filter predicts the person's position as a candidate sample for the detector. Then, the designed FZ-IGS is used to change the turning gain and linear velocity of the robot according to the position of the person from the robot. The FZ-IGS drives the robot towards the person continuously and stably. A conventional method for automated guided vehicle (AGV) localization has certain limitations, such as slip phenomena, because there are variations in the surface of the road and ground friction. Therefore, precise localization is a very important issue for the inevitable slip phenomenon situation. The paper by S.-W. Yoon et al. presents a sensor fusion method to cope with this drawback by using the Kalman filter, which can eliminate the disadvantages of each sensor, such as the image sensor and encoder based sensor, and obtains the precise localization of the AGV in a slip phenomenon situation.

\section{Acknowledgments}

At last, we would like to thank all the authors for their valuable contributions and also the reviewers for their critical help, necessary to achieve a high level of papers' quality and thus make the completion of this special issue possible. Also we would like to thank all members of Editorial Board for approving this special issue.

$$
\begin{array}{r}
\text { Aiguo Song } \\
\text { Guangming Song } \\
\text { Daniela Constantinescu } \\
\text { Lei Wang } \\
\text { Quanjun Song }
\end{array}
$$



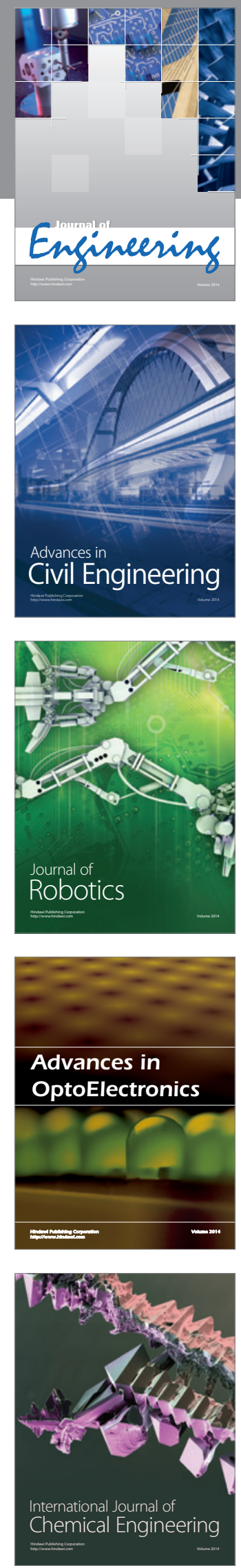

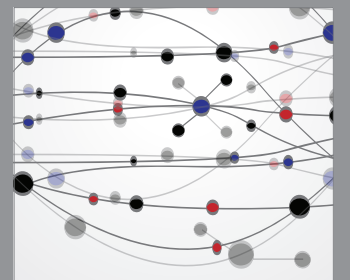

The Scientific World Journal
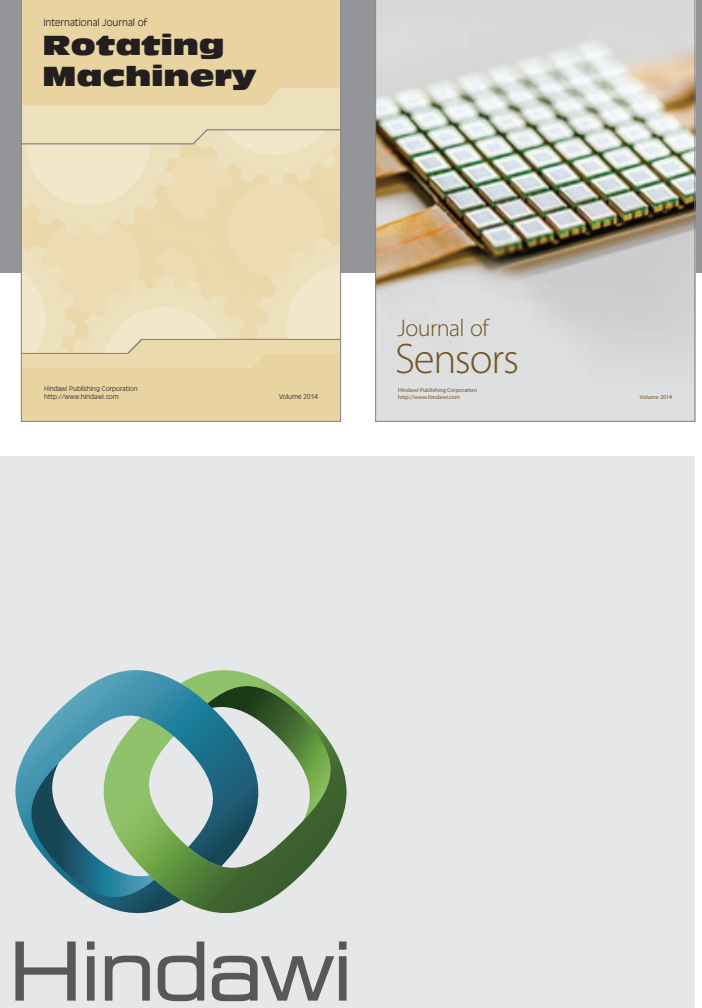

Submit your manuscripts at http://www.hindawi.com
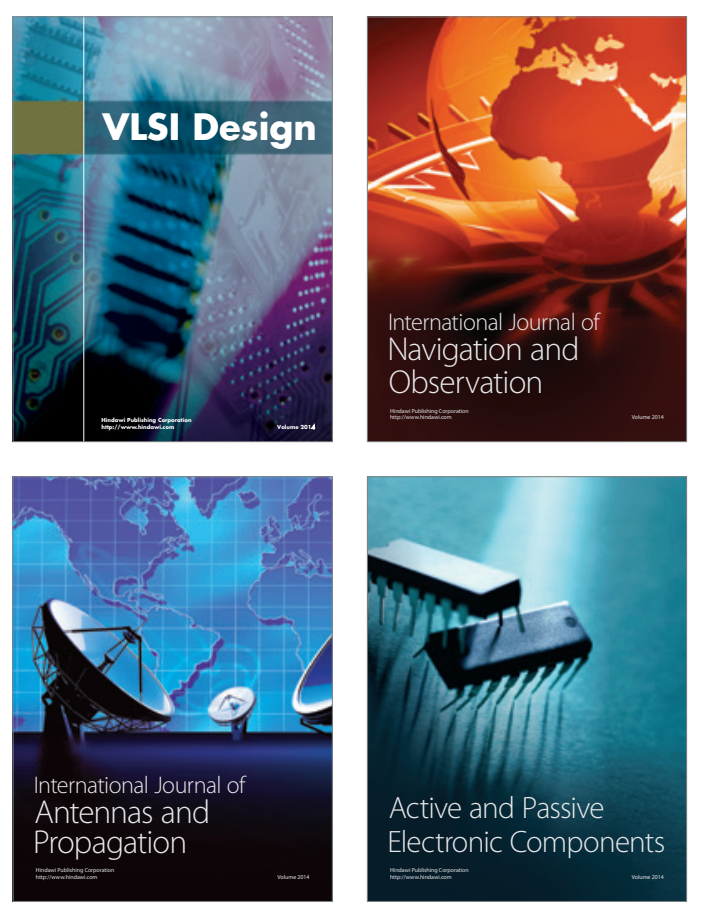
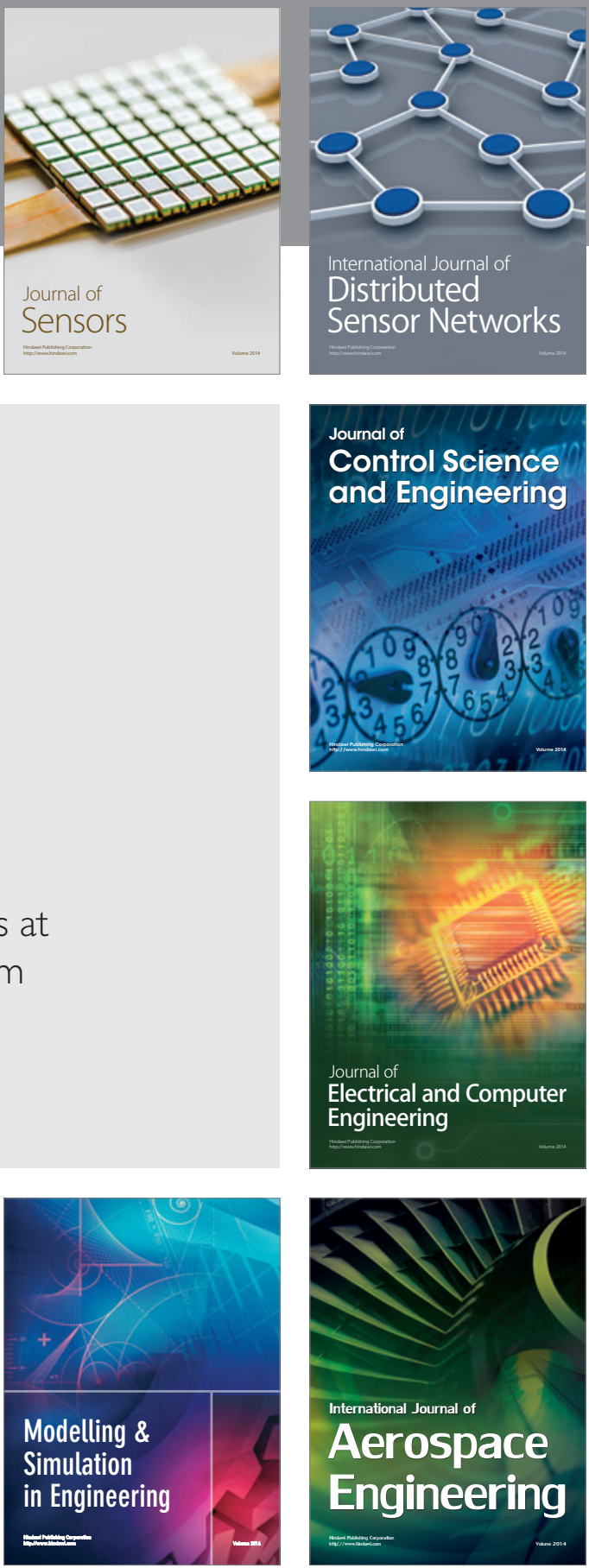

Journal of

Control Science

and Engineering
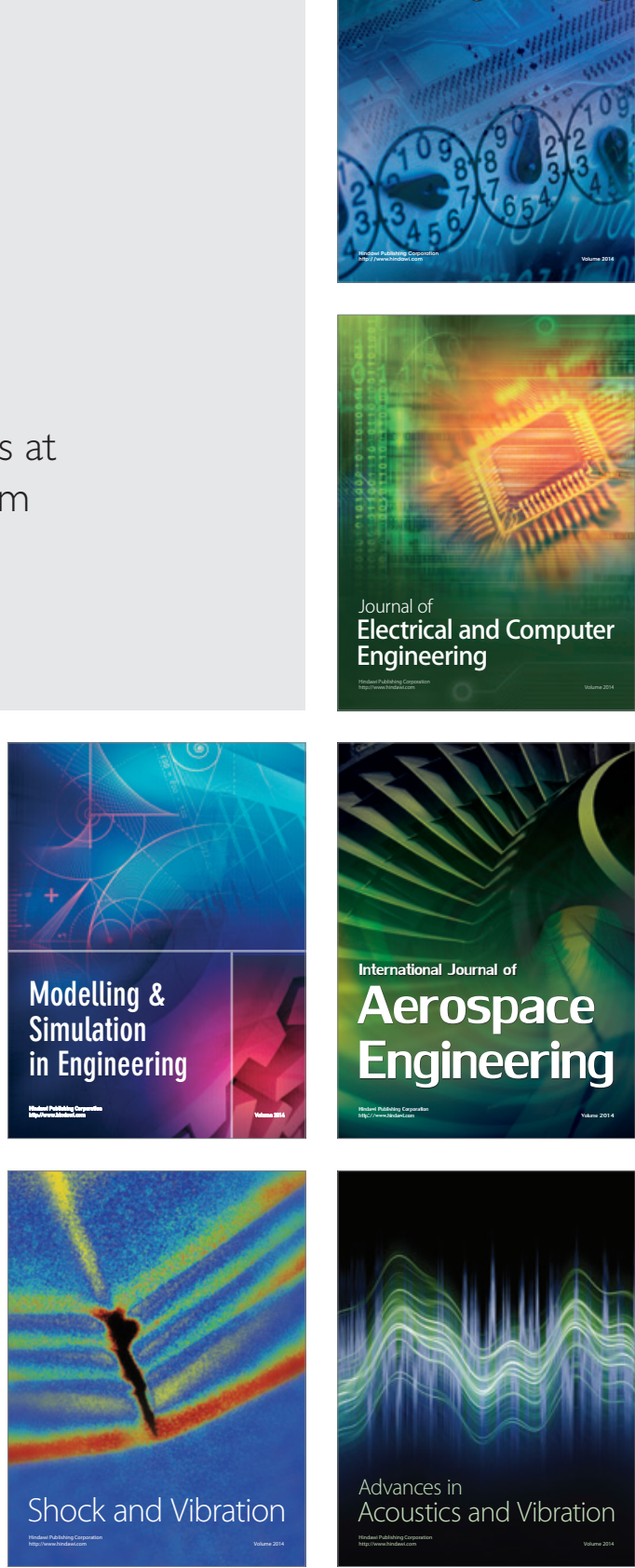